\title{
Breast Myofibroblastoma
}

National Cancer Institute

\section{Source}

National Cancer Institute. Breast Myofibroblastoma. NCI Thesaurus. Code C40397.

A myofibroblastoma occurring in the breast of both women and men. It presents as a slowly growing mass. 